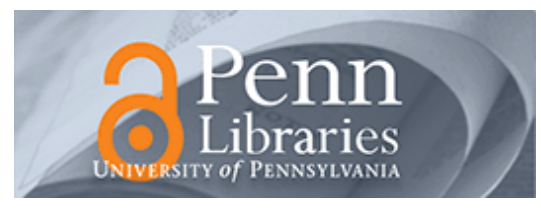

University of Pennsylvania ScholarlyCommons

9-30-1991

\title{
Atomic Structure of a Grain Boundary in a Metallic Alloy: Combined Electron Microscope and Theoretical Study
}

David E. Luzzi

University of Pennsylvania, luzzi@Irsm.upenn.edu

Min Yan

Mojmír Šob

Vaclav Vitek

University of Pennsylvania, vitek@seas.upenn.edu

Follow this and additional works at: https://repository.upenn.edu/mse_papers

Part of the Engineering Physics Commons, and the Structural Materials Commons

\section{Recommended Citation}

Luzzi, D. E., Yan, M., Šob, M., \& Vitek, V. (1991). Atomic Structure of a Grain Boundary in a Metallic Alloy: Combined Electron Microscope and Theoretical Study. Physical Review Letters, 67 (14), 1894-1897. http://dx.doi.org/10.1103/PhysRevLett.67.1894

This paper is posted at ScholarlyCommons. https://repository.upenn.edu/mse_papers/231

For more information, please contact repository@pobox.upenn.edu. 


\title{
Atomic Structure of a Grain Boundary in a Metallic Alloy: Combined Electron Microscope and Theoretical Study
}

\author{
Abstract \\ A synergistic high-resolution electron microscopy (HREM) and theoretical analysis of the structure of a \\ grain boundary in copper containing bismuth is presented. The calculation of the structure of the \\ boundary were carried out using $\mathrm{N}$-body empirical potentials constructed using results of $a b$ initio full- \\ potential linear-muffin-tin-orbital calculations. Excellent agreement between the calculated and observed \\ structures is shown by comparing a through-focal series of observed and calculated images. It is shown \\ for the first time that HREM combined with computer modeling employing realistic empirical potentials \\ can decipher with a great accuracy the structure of boundaries containing multiple atomic species. \\ Disciplines \\ Engineering | Engineering Physics | Materials Science and Engineering | Structural Materials
}




\title{
Atomic Structure of a Grain Boundary in a Metallic Alloy: Combined Electron Microscope and Theoretical Study
}

\author{
D. E. Luzzi, Min Yan, M. Šob, ${ }^{(a)}$ and V. Vitek \\ Department of Materials Science and Engineering, University of Pennsylvania, Philadelphia, Pennsylvania 19104-6272 \\ (Received 17 April 1991)
}

\begin{abstract}
A synergistic high-resolution electron microscopy (HREM) and theoretical analysis of the structure of a grain boundary in copper containing bismuth is presented. Calculations of the structure of the boundary were carried out using $N$-body empirical potentials constructed using results of $a b$ initio fullpotential linear-muffin-tin-orbital calculations. Excellent agreement between the calculated and observed structures is shown by comparing a through-focal series of observed and calculated images. It is shown for the first time that HREM combined with computer modeling employing realistic empirical potentials can decipher with great accuracy the structure of boundaries containing multiple atomic species.
\end{abstract}

PACS numbers: $61.70 . \mathrm{Ng}, 07.80 .+\mathrm{x}, 68.35 . \mathrm{Bs}, 68.35 . \mathrm{Dv}$

The atomic structure of grain boundaries in metallic materials, in particular in alloys, is the key to understanding a wide variety of their physical mechanical properties. Until recently, most of such information was obtained either from indirect observations, such as the investigation of intergranular fracture surfaces, or from computer simulations based on model interatomic potentials [1,2]. For some special grain boundaries structural information has been acquired using $\mathrm{x}$-ray diffraction [3-7] which has been a ubiquitous approach to probing crystal structures of elements and compounds. Notwithstanding, this method is difficult to apply to interfaces since the strength of the signal from these regions is extremely small and the determination of exact atomic positions is not unambiguous [6-8].

On the other hand, high-resolution electron microscopy (HREM) relies upon the strong scattering of electrons and direct detailed information on the structure of material volumes of atomic dimensions is thus accessed. However, a firm interpretation of the HREM images can only be made if a physically reliable model of the studied structure can be established and a direct comparison of observed and theoretically calculated images made. This combined experimental and theoretical approach is then synergistic in that the "raw image" suggests the best first approximation of the structure studied and the fully relaxed structures provide a guide for the best choice of the imaging conditions. An essential prerequisite of theoretical investigations is a reliable evaluation of the total energy of the system studied as a function of atomic position. While in the previous studies of the atomic structures generic interatomic potentials were often sufficient $[1,2,9]$, dependable descriptions of atomic interactions for specific material systems is essential since changes in atomic positions as small as $0.1 \AA$ invoke substantial variations in the image.

Recently, such a synergistic approach to the characterization of grain-boundary structures has been applied in pure metals $[10,11]$. However, it has never been employed in the case of alloys where the aim is not only to decipher the atomic positions but also to determine the chemical nature of the atoms. In this paper we report the first such combined HREM and theoretical study for the case of a grain boundary in copper containing a high concentration of segregated bismuth. The boundary is the $\Sigma=3(111) /(11 \overline{1})$ boundary formed by the reversible faceting induced by the segregation of $\mathrm{Bi}$ [12-14]. In pure copper this is a symmetrical tilt boundary with the rotation axis $[1 \overline{1} 0]$, i.e., the twin boundary. Such boundaries are ideally suited for HREM analysis since the boundary plane does not deviate from the $\left\{\begin{array}{lll}1 & 1\end{array}\right\}$ plane and it can thus be maintained parallel to the electron beam. The calculation of the boundary structure has been made using $N$-body potentials of the Finnis-Sinclair type [15], analogous to the embedded-atom method [16]. These were constructed using the approach proposed by Ackland and Vitek [17], and with the help of $a b$ initio electronic structure calculations employing the full-potential linear-muffin-tin-orbital method [18].

The HREM analysis has been performed using a JEOL JEM 4000EX microscope. The sample containing the above-mentioned boundary was tilted such that the electron beam was aligned along the [1 10$]$ direction which is a common crystal direction for both grains. Images were then recorded at several values of objective-lens defocus. From the systematic variation of the image contrast with defocus we can extract information on both the positions and identities of the atoms [19]. In general, this involves an iterative process in which calculated images of model structures are compared to the experimental images. A series of experimental images of the boundary studied is presented in the left column of Fig. 1. When interpreting these images, many model structures, commensurate with the observed periodicities within the interface, were first constructed geometrically, without any considerations of the energy of the system. Comparison with the experiment was then made by calculating images arising from these models as a function of defocus using the multislice formalism. The model structure which led to the best match of calculated and observed images can 


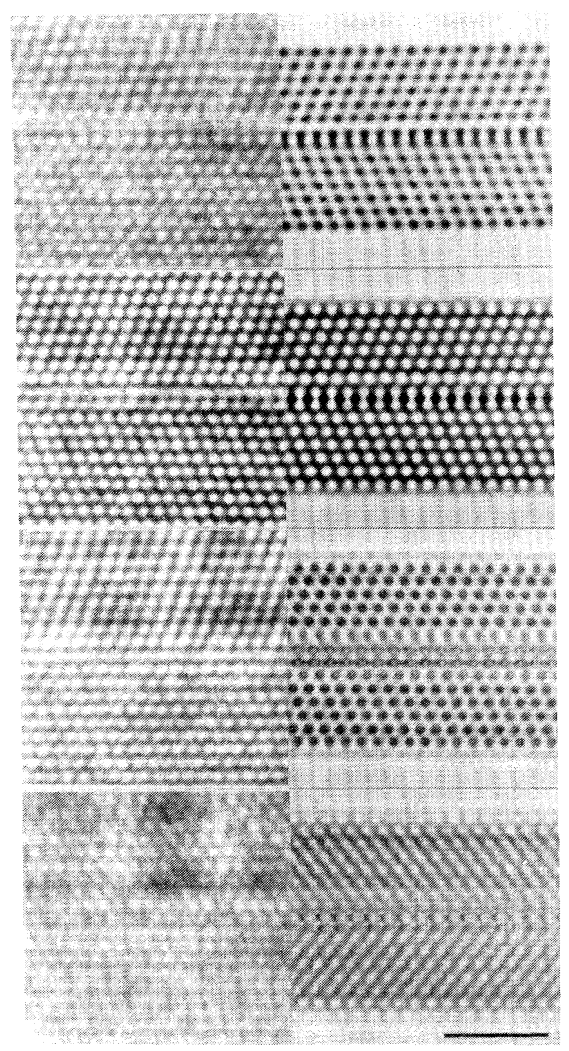

FIG. 1. Experimental (left column) and calculated (right column) image series with the objective-lens underfocus increasing down the figure. The marker represents $15 \AA$.

be described as follows. In the framework of the " $A B C$ " description of the sequence of $\{111\}$ atomic planes in an fcc crystal, the $\Sigma=3(111) /(11 \overline{1})$ twin in pure copper can be represented as

$$
A-B-C-A-B-\stackrel{\mid}{C}-B-A-C-B-A-C,
$$

where the vertical lines mark the position of the boundary. The model structure could be represented in the same way with the marked layer $C$ denoting a $\{111\}$ plane in which one-third of the copper atoms were replaced by hexagonally arranged $\mathrm{Bi}$ atoms. However, owing to their size, the $\mathrm{Bi}$ atoms are centered outside this atomic plane so that a physically more appropriate representation of the model structure is

$$
A-B-C-A-B-C^{\prime} \frac{1}{\Gamma} C(\mathrm{Bi})-B-A-C-B-A-C,
$$

where $C^{\prime}$ denotes a plane of copper atoms in which onethird of the atoms were replaced by hexagonally arranged vacancies, and $C(\mathrm{Bi})$ denotes the plane of bismuth atoms positioned above these vacancies.

In order to determine if this structure is stable, and, if so, to find the actual location of the bismuth atoms within the boundary and the rigid-body expansion, atomistic computer modeling of this boundary was carried out.
The same method as in a number of previous studies of the grain-boundary structures has been employed. A block of (copper) atoms, containing in the middle the $\Sigma=3(111) /(11 \overline{1})$ boundary, was first constructed geometrically. The boundary region was then modified by replacing some of the copper atoms by bismuth atoms so as to form the structure described above. This structure was then used as a starting configuration in the relaxation calculation in which the total energy of the block has been minimized with respect to the positions of the individual atoms as well as with respect to the relative rigid-body displacement of the two grains. This displacement has components both parallel and perpendicular to the boundary so that there are neither any tensile nor compressive stresses normal to the boundary nor shear stresses parallel to the boundary present in the final configuration. The displacement perpendicular to the boundary represents the expansion. The periodic boundary conditions in the directions parallel to the boundary have been maintained throughout the calculation while in the direction perpendicular to the boundary the block is effectively infinite but it was found that it was sufficient to limit the relaxation to $22\{111\}$ layers on each side of the boundary.

The total energy of the system of $N$ atoms is written in the present work as in the Finnis-Sinclair scheme $[15,20,21]$,

$$
E=\frac{1}{2} \sum_{i, j=1}^{N} V_{T_{i} T_{j}}\left(R_{i j}\right)-\sum_{i=1}^{N}\left(\sum_{j} \phi_{T_{i} T_{j}}\left(R_{i j}\right)\right)^{1 / 2}
$$

where $V_{T_{i} T_{j}}$ and $\phi_{T_{i} T_{j}}$ are pair potentials, generally dependent on species $T_{i}$ and $T_{j}$, and $R_{i j}$ is the separation of atoms $i$ and $j$. The $\mathrm{Cu}-\mathrm{Cu}$ potentials were fitted so as to reproduce the experimentally known density, cohesive energy, elastic constants, and vacancy formation energy of pure copper [20]; the $\mathrm{Bi}-\mathrm{Bi}$ potentials reproduce the density and the cohesive energy of the high-pressure bcc phase of bismuth. $\phi_{\mathrm{Cu}-\mathrm{Bi}}$ has been taken as the geometrical mean of the $\phi$ potentials for $\mathrm{Cu}$ and $\mathrm{Bi}$, respectively. However, the pair potential $V_{\text {Cu-Bi }}$ has been fitted so as to reproduce the equilibrium density, the bulk modulus, and the shear modulus $C_{11}-C_{12}$ calculated for a hypothetical $L 1_{2}$ compound $\mathrm{Cu}_{3} \mathrm{Bi}$. This calculation was performed using the all-electron self-consistent full-potential linearmuffin-tin-orbital method within the local-density approximation [18]. The potentials also reproduce the experimentally measured enthalpy of mixing of the liquid $\mathrm{Cu}$ Bi solution at $1200 \mathrm{~K}$. The details of the construction of these $N$-body potentials are presented in Ref. [22].

The relaxation calculations, indeed, show that stable structures similar to that described above, and used as the starting configuration, exist. Two such structures, corresponding to different sizes of the repeat cell of the boundary, have been found. The lowest-energy structure has been obtained for the repeat cell $\frac{3}{2}[1 \overline{1} 0] \times[11 \overline{2}]$ and also when using the repeat cell $3[1 \overline{1} 0] \times 2[11 \overline{2}]$. On the other hand, the energy of the structure with the smallest possi- 
ble repeat cell, $\frac{3}{2}[1 \overline{1} 0] \times \frac{1}{2}[11 \overline{2}]$, is about $1.5 \%$ higher. The low-energy structure of the boundary studied is shown in Fig. 2 projected onto the (110) plane. The copper atoms are represented by small circles and the bismuth atoms by large circles. The four different symbols, crosses, squares, circles, and triangles, represent, in this order, atoms belonging to four subsequent (1ī0) planes separated by the distance $\left|\frac{1}{4}[1 \overline{1} 0]\right|$; only four rather than six different $(1 \overline{1} 0)$ planes are shown for clarity. In this structure the overall expansion of the bicrystal, caused by the rigid-body displacement of the two grains across the boundary, is $2.09 \AA$. This is very close to the interplanar spacing of $\{111\}$ planes in $\mathrm{Cu}$ and thus it could be interpreted as the splitting of one of the $\{111\}$ planes, $C$, into two, $C^{\prime}$ and $C(\mathrm{Bi})$, containing $\mathrm{Cu}$ and $\mathrm{Bi}$, respectively. However, this splitting does not produce simply two $\{111\}$ planes spaced as in copper. Because of the structural relaxation the plane of bismuth atoms [C $(\mathrm{Bi})]$ is contracted towards the plane of copper containing vacancies $\left(C^{\prime}\right)$ by $0.5 \AA$ away from the ideal position, while the overall expansion is spread across several $\{111\}$ layers.

In order to make a detailed comparison of the calculated and observed structures, a series of images, corresponding to the same defocus conditions and thickness as in the HREM study, were calculated for the low-energy relaxed structure using the multislice formalism. The thickness and defocus values were established based on the images of the $\mathrm{Cu}$ grains away from the interface (see Ref. [19] for details). The calculated images are presented in the right column of Fig. 1. The following are the most important features of the images which are clearly visible in both the experimental and calculated images. In the first image, the boundary consists of a centered row of discrete light contrast with a slight asymmetry of the contrast of the first pure $\mathrm{Cu}$ planes bounding the interface. In the second image, the boundary appears as a ladder structure with streaking present between the atom contrast within the interface. In the third image, the boundary again consists of light contrast centered in the boundary although it tends to be more streaked than in the first image. In the fourth image, it is seen that the (200) fringes terminate adjacent to dark contrast in the boundary.

This degree of match in the image series is strong evidence for the very high accuracy with which the structure has been predicted theoretically. Furthermore, the calculated expansion, i.e., the rigid-body displacement of the two grains across the boundary, is, within the experimental limits of the accuracy in the HREM $(\approx 0.1 \AA)$, in perfect agreement with the experiment. When considering the contraction of the bismuth-atom plane towards the copper plane containing vacancies a somewhat better match between the observed and calculated images is obtained for a contraction of $0.4 \AA$ than for the predicted contraction of $0.5 \AA$. Nevertheless, this extent of con-

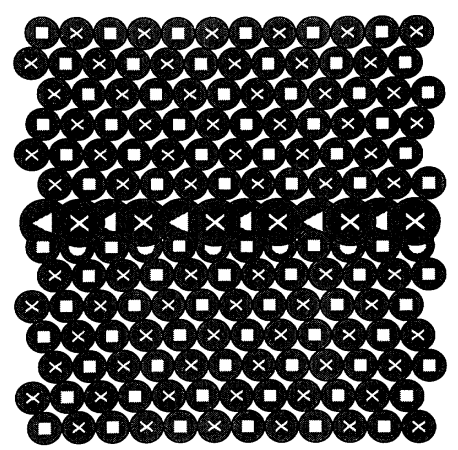

FIG. 2. Calculated structure of the $\Sigma=3(111) /(11 \overline{1})$ symmetrical tilt boundary containing bismuth.

currence between the calculated and the experimentally imaged structures is remarkable.

The results presented here demonstrate for the first time that HREM studies and theoretical structural analyses can be used synergistically to decipher with a great accuracy the structure of boundaries containing multiple atomic species. At the same time these results provide firm evidence that the empirical $N$-body potentials are capable of describing with a sufficient accuracy atomic interactions even in relatively complex systems such as $\mathrm{Cu}-\mathrm{Bi}$ provided an appropriate and sufficient input is used in their construction. For this purpose the self-consistent $a b$ initio electronic structure calculations may provide data corresponding to sampling of configurations which are not attainable experimentally. This approach has been employed in the present study where it was a crucial step in determining appropriate $N$-body potentials for the copper-bismuth system. These potentials, validated by the comparison with the HREM results, can now be used with a high confidence to investigate the structure of grain boundaries which are experimentally inaccessible, as well as other atomic level processes such as bismuth diffusion and segregation in copper.

The authors are grateful for the use of the University of Pennsylvania MRL central facilities and would like to thank D. A. Ricketts-Foot for maintaining the microscope and B. Blum for preparing the specimens. This research was supported by the National Science Foundation, Materials Research Laboratories Program DMR88-19885 (D.E.L.) and by the U.S. Department of Energy, Office of Basic Energy Sciences, Grant No. DEFG02-87ER45295 (M.Y., M. S., and V.V.).

(a) On leave from the Institute of Physical Metallurgy, Czechoslovak Academy of Sciences, Zizkova 22, Brno, Czechoslovakia.

[1] P. D. Bristowe, in Proceedings of the Fourth Japan Institute of Metals International Symposium on Grain Boundary Structure and Related Phenomena [Trans. Jpn. Inst. Metals 27, 89 (1986)]. 
[2] A. P. Sutton, in Proceedings of the International Congress on Intergranular and Interphase Boundaries in Materials [J. Phys. (Paris), Colloq. 51, C1-35 (1990)].

[3] S. L. Sass, J. Appl. Crystallogr. 13, 109 (1980).

[4] J. Budai, P. D. Bristowe, and S. L. Sass, Acta Metall. 31, 699 (1983).

[5] M. R. Fitzsimmons and S. L. Sass, Acta Metall. 37, 1009 (1989).

[6] M. S. Taylor, I. Majid, P. D. Bristowe, and R. W. Balluffi, Phys. Rev. B 40, 2772 (1989).

[7] I. Majid, P. D. Bristowe, and R. W. Balluffi, Phys. Rev. B 40, 2779 (1989).

[8] Y. Oh and V. Vitek, Acta Metall. 34, 1941 (1986).

[9] V. Vitek and J. Th. M. DeHosson, in Computer-Based Microscopic Description of the Structure and Properties of Materials, edited by J. Broughton, W. Krakow, and S. T. Pantelides, MRS Symposia Proceedings No. 63 (Materials Research Society, Pittsburgh, 1986), p. 125.

[10] U. Dahmen, C. J. D. Hetherington, M. A. O’Keefe, K. H. Westmacott, M. J. Mills, M. S. Daw, and V. Vitek, Philos. Mag. Lett. 62, 327 (1990).

[11] M. J. Mills and M. S. Daw, in High Resolution Electron Microscopy of Defects in Materials, edited by R. Sinclair, D. J. Smith, and U. Dahmen, MRS Symposia Proceedings No. 183 (Materials Research Society, Pittsburgh, 1991), p. 15.
[12] T. G. Ference and R. W. Balluffi, Scr. Metall. 22, 1929 (1988).

[13] M. Menyhard, B. Blum, and C. J. McMahon, Jr., Acta Metall. 37, 549 (1989).

[14] B. Blum, M. Menyhard, D. E. Luzzi, and C. J. McMahon, Jr., Scr. Metall. 24, 2169 (1990).

[15] M. W. Finnis and J. E. Sinclair, Philos. Mag. A 50, 45 (1984).

[16] M. S. Daw and M. I. Baskes, Phys. Rev. B 29, 6443 (1984).

[17] G. J. Ackland and V. Vitek, Phys. Rev. B 41, 10324 (1990).

[18] M. Methfessel, C. O. Rodriguez, and O. K. Andersen, Phys. Rev. B 40, 2009 (1989).

[19] D. E. Luzzi, in Proceedings of the Twelfth International Congress for Electron Microscopy, edited by L. D. Peachey and D. B. Williams (San Francisco Press, San Francisco, 1990), p. 318; Ultramicroscopy (to be published).

[20] G. J. Ackland, G. Tichy, V. Vitek, and M. W. Finnis, Philos. Mag. A 56, 735 (1987).

[21] G. J. Ackland, M. W. Finnis, and V. Vitek, J. Phys. F 18, L153 (1988).

[22] Min Yan, G. J. Ackland, M. Sob, V. Vitek, M. Methfessel, C. O. Rodriguez, and O. K. Andersen (to be published). 


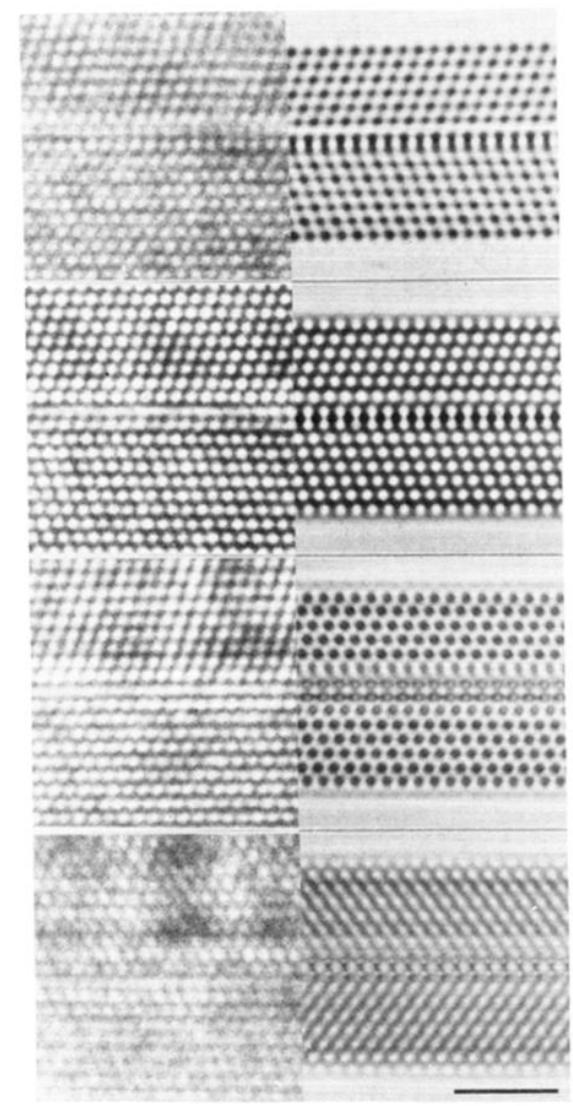

FIG. 1. Experimental (left column) and calculated (right column) image series with the objective-lens underfocus increasing down the figure. The marker represents $15 \AA$. 